\title{
EDITORIAL
}

\section{Proteolytic enzymes and airway diseases}

\author{
J.A. Nadel*, R.A. Stockley+
}

For some 30 yrs proteolytic enzymes have been implicated in the pathogenesis of destructive lung diseases, with particular emphasis on their role in the development of emphysema. However, in recent years our concepts and views about proteolytic enzymes have increased dramatically. It is now recognized that some enzymes are exquisitely selective in their substrate specificities and may play a role in the development of many features of lung disease, particularly in the airway. In view of this rapidly expanding field of knowledge it was thought to be timely to organize a symposium on several new aspects of proteinases and lung disease, which took place at the 1997 ERS Annual Congress. Nevertheless, since the topic is expanding so rapidly it was felt necessary to be selective in the choice of subjects for the symposium. They were chosen predominantly to cover areas of current research interest, to include subjects from diverse areas of respiratory disease and to give priority to areas of particular clinical relevance.

The symposium covered four topics, which are now being published as a series of reviewed articles in the journal.

\section{Neutrophil serine proteinases and defensins in chronic obstructive pulmonary disease}

HIEMSTRA et al. [1] reviewed the evidence for the role of neutrophils in the pathogenesis of chronic obstructive pulmonary disease (COPD). In particular, some of the neutrophil enzymes, such as elastase, cathepsin G, proteinase 3 that have long been viewed as important in both the interstitium of the lung and the airways were described. In addi-tion, the role of defensins, which are also present in the azurophil granule of the neutrophil and have been shown to mediate epithelial damage was emphasized. HIEMSTRA et al. [1] discussed in detail the interaction between proteinases, their natural inhibitors and the role played by in-flammatory cell-derived oxidants in the disturbance of the proteinase/antiproteinase balance in the lung. The experiments showing the relative contribution of defensins and serine proteinases to neutrophil-mediated lung injury were described. In particular, this review focused on the contribution of defensins, not only to the efficient intracellu- lar antimicrobial action of neutrophils but also the way in which the extracellular activity of these potent proteins might play a role in COPD. This latter role includes

*Cardiovasc Research Institute, San Francisco, CA, USA. +Dept of Medicine, Queen Elizabeth Hospital, Birmingham, B15 2TH, UK.

Correspondence: J.A. Nadel, University of California, Cardiovasc Research Institute, 505 Parnassus Avenue., Box 0130, San Francisco, CA 95143-0130, USA. Fax: 14154762283 not only damage to the bronchial epithelium itself, but also the ability to stimulate epithelial cells to produce neutrophil chemoattractants, thereby, amplifying the inflammatory pro-cess.

\section{Neutrophil endopeptidase}

Neutrophil endopeptidase (NEP) is a metallopeptidase discovered on the brush border epithelium of the kidney. It is a highly selective peptidase, hydrolysing peptide bonds of substances and other peptides yielding inactive fragments. Di Maria et al. [2] in this issue of the Journal discusses the actions of NEP in the modulation of neurogenic inflammation. In particular, the authors cover the distribution of NEP in the lung, its role in inactivating kinins, and the possible effect on lung disease.

\section{The role of neutrophil elastase in hypersecretion in asthma}

Bronchospasm is widely recognized as the major cause of symptoms in asthma. However, it is less well recognized that hypersecretion of mucus plays an important role in acute, severe asthma and that mucus plugging, especially in the peripheral airways, is a major finding in post mortem studies of individuals who die of acute asthma. NADEL et al. [3] present results implicating neutrophils and neutrophil elastase, in particular in mucus hypersecretion in asthma. The authors present data suggesting that the neutrophil-dependent goblet cell secretion involves tight adhesion between goblet cell receptors (intercellular adhesion molecule-1 (ICAM-1)) and neutrophil ligands (CD11b/ CD18). This tight adhesion permits elastase activity to occur at the interface between the cells, leading to dramatic and complete degranulation of the goblet cell. These novel findings are shown to occur in human airways and this raises the concept of novel therapeutic approaches for the prevention of mucus secretion and airway plugging.

\section{Mast cell tryptase}

Mast cell tryptase is an enzyme that is manufactured and released by mast cells. It has received major attention recently because of its abundance and its value as an in vivo marker of mast cell activation. It has several actions which are also compatible with a role in asthmatic inflammation. WALLS [4] discusses the cellular distribution and physicochemical and enzyme properties of tryptase as well as other mast cell enzymes. The author points to the diverse functions of tryptase on many factors that could cause and enhance the inflammatory process within the lung. In addition, data is provided showing that tryptase is also a 
potent growth factor for lung cells and may therefore play a role in tissue remodelling. More recent data indicates that inhibitors of tryptase have been shown to influence the clinical features of allergen-induced bronchoconstriction, providing the potential for new therapeutic approaches in the future.

\section{References}

1. Hiemstra PS, Van Wetering S, Stolk J. Neutrophil serine proteinases and defensins in chronic obstructive pulmo- nary disease: effects on pulmonary epithelium. Eur Respir J 1998; 12: 1200-1208.

2. Di Maria GU, Bellofiore S, Geppetti P. Regulation of airway neurogenic inflammation by neutral endopeptidase. Eur Respir J 1998; 12: 1454-1462.

3. Nadel JA, Takeyama K, Agusti C. Role of neutrophil elastase in hypersecretion in asthma. Eur Respir J 1999; 13: in press.

4. Walls AF. Mast cell tryptase and chymase as major mediators in airway inflammation. Eur Respir J 1999; 13: in press. 